\title{
Convective boundary-layer height estimation from combined radar and Doppler lidar observations in VORTEX-SE
}

Villalonga, Joan, Beveridge, Susan, Araujo da Silva, Marcos, Tanamachi, Robin, Rocadenbosch, Francesc, et al.

Joan Villalonga, Susan L. Beveridge, Marcos P. Araujo da Silva, Robin L. Tanamachi, Francesc Rocadenbosch, David D. Turner, Stephen J. Frasier, "Convective boundary-layer height estimation from combined radar and Doppler lidar observations in VORTEX-SE," Proc. SPIE 11531, Remote Sensing of Clouds and the Atmosphere XXV, 115310X (20 September 2020); doi: $10.1117 / 12.2576046$

Copyright 2020 Society of Photo Optical Instrumentation Engineers. One print or electronic copy may be made for personal use only. Systematic electronic or print reproduction and distribution, duplication of any material in this paper for a fee or for commercial purposes, or modification of the content of the paper are prohibited.

http://dx.doi.org/10.1117/12.2576046 


\title{
Convective Boundary-Layer Height Estimation from Combined Radar and Doppler Lidar Observations in VORTEX-SE
}

\author{
Joan Villalonga ${ }^{1}$, Susan L. Beveridge ${ }^{2}$, Marcos P. Araujo da Silva ${ }^{1}$, Robin L. Tanamachi ${ }^{2}$, \\ Francesc Rocadenbosch ${ }^{1,3}$, David D. Turner ${ }^{4}$, and Stephen J. Frasier ${ }^{5}$ \\ ${ }^{1}$ CommSensLab-UPC, Department of Signal Theory and Communications, Universitat \\ Politècnica de Catalunya (UPC), C/ Jordi Girona, 1-3, E-08034, Barcelona, Spain. \\ ${ }^{2}$ Dept. of Earth, Atmospheric, and Planetary Sciences, Purdue University, 550 West Stadium \\ Avenue, West Lafayette, IN 47906 (USA). \\ ${ }^{3}$ Institut d'Estudis Espacials de Catalunya (Institute of Space Studies of Catalonia, IEEC), \\ Barcelona, Spain, E-08034 Barcelona, Spain \\ ${ }^{4}$ Global Systems Laboratory / Earth System Research Laboratories / NOAA, 325 Broadway, \\ Boulder, CO 80305-3337 (USA). \\ ${ }^{5}$ Microwave Remote Sensing Laboratory, University of Massachusetts, 151 Holdsworth Way, \\ Suite 113, Amherst, MA 01003 (USA).
}

\begin{abstract}
The Verification of the Origins of Rotation in Tornadoes Experiment - Southeast (VORTEX-SE) provides a wealth of long-duration, high-resolution, vertically pointing observations from active and passive ground-based remote sensing systems enabling characterization of the Atmospheric Boundary Layer (ABL) development over distinct regions that are well known for their relatively high tornado frequency. Application of the Extended Kalman Filter (EKF) to BL height estimation in the convective regime (CBLH) of the diurnal cycle from S-band radar reflectivity observations ${ }^{1}$ has shown to yield accurate results under simple CBL conditions. In this work, we revisit the radar-EKF technique and investigate its main limitations. For example, during daytime clear-sky conditions such as those prevailing in the BL morning transition, weak turbulence leads to very low reflectivity returns, limiting application of this technique. Additionally, turbulent mixing layers capped with a residual layer, and/or multi-layer scenarios can lead the filter to lose track of the BL signature over time. Doppler Wind Lidar (DWL) observations of the vertical wind velocity variance ${ }^{2}$ provide complementary CBLH estimates to those of the radar-EKF combination, providing potential to disambiguate more complex convective cases. DWL estimates are, however, strongly influenced by the variance threshold selected. The complementarity of radar and DWL for CBLH estimation is studied in reference to radiosoundings.
\end{abstract}

Keywords: atmospheric boundary layer, S-band radar, Doppler lidar, adaptive filtering, estimation.

\section{INTRODUCTION}

During daytime over land, the Earth's surface is generally warmer than the air and, as a consequence, the convective turbulence acts strongly to vertically mix the air within the atmospheric boundary layer (ABL). ${ }^{3} \mathrm{In}$ this condition, the ABL is called the "mixed layer" (ML) or convective boundary layer (CBL). ${ }^{3,4}$

The estimate of the CBL height (CBLH) with high spatio-temporal resolution is a matter of interest with regard to pollutant dispersion, general weather forecasting, and tornado potential. ${ }^{3}$ The Verification of the Origins of Rotation in Tornadoes EXperiment - Southeast (VORTEX-SE) project aimed to investigate tornado

Further author information: (Send correspondence to F.R. and R.T.)

F.R.: E-mail: roca@tsc.upc.edu, Telephone: +34 934054114

R.T.: E-mail: rtanamachi@purdue.edu, Telephone: +1 (765) 496-2866

Remote Sensing of Clouds and the Atmosphere XXV, edited by Adolfo Comerón, Evgueni I. Kassianov, Klaus Schäfer, Richard H. Picard, Konradin Weber, Upendra N. Singh, Proc. of SPIE Vol. 11531,

115310X - (c) 2020 SPIE · CCC code: 0277-786X/20/\$21 - doi: 10.1117/12.2576046 
formation, evolution, and impacts in the Southeast United States. ${ }^{5}$ In order to achieve these objectives, two multi-week spring measurement campaigns were carried out in 2016 and 2017 in Northern Alabama. Radar, radiosonde, Doppler Wind Lidar (DWL), and Atmospheric Emitted Radiance Interferometer (AERI) were used to intensively monitor the ABL (See Ref. 6 for details). In this work, a vertically pointing S-band Frequency Modulated Continuous Wave (FMCW) radar was combined with an extended Kalman filter (EKF) to retrieve CBLH estimates under clear-day non-precipitating conditions with a root-mean-square difference of about 170 $m$ when compared to AERI-derived thermodynamic profiles. ${ }^{6}$ The EKF can be understood as an adaptive morphological filter tracking the abrupt CBL-to-free-atmossphere (FA) transition. The reader is referred to Ref. 1 for foundations of the EKF technique when applied to time series of radar reflectivity profiles, and Ref. 7 when applied to time series of backscatter lidar returns. The morphological bases for this technique can, however, lead to incorrect CBLH height estimates in weak turbulence conditions such as those prevailing in the morning and afternoon transition periods, or to lead the filter to track the residual layer $(\mathrm{RL})^{3}$ left over from the preceding days' CBL.

In this study we evaluate the CBLH estimation performance of the radar-EKF combination against two collocated instruments, the DWL and the AERI, which are part of the Collaborative Lower Atmospheric Mobile Profiling System (CLAMPS-2). The present manuscript is has a companion paper ${ }^{8}$ devoted to ABL height monitoring during different periods in the ABL diurnal cycle. This work is structured as follows: Sect. 2 describes the instruments used in VORTEX-SE field campaign, Sect. 3 explains the CBLH retrieval methods. Sect. 4 discusses a case study, and shows global statistics encompassing 19 days of the VORTEX-SE 2017 campaign. Finally, Sect. 5 gives concluding remarks.

\section{VORTEX-SE INSTRUMENTS}

During the VORTEX-SE field campaign, all the instruments in this study were deployed in Scottsboro Airport, Alabama (latitude: $34.68728{ }^{\circ} \mathrm{N}$, longitude: $86.00508{ }^{\circ} \mathrm{W}$ ), from 10 March to 1 May 2017. A total of 19 days were selected to investigate the CBL period, corresponding to cloud- and precipitation-free days in order to avoid outliers. A Vaisala CL31 ceilometer, which is a low cost lidar, was used to retrieve the estimates of cloud base height $(\mathrm{CBH})$. The set of instruments included the S-band, $2.92 \mathrm{GHz}$, FMCW radar developed by the University of Massachusetts (hereafter, UMass S-band radar), which is a transportable, pulse-compression, single-polarized radar capable of automatically monitoring the ABL through vertically-pointed measurements. ${ }^{9}$ Table 2 summarizes the main specification of the instruments.

The CLAMPS-2 unit $^{10}$ is a compact, mobile ABL monitoring system mounted inside of a 4.9-m long trailer. During VORTEX-SE, it facilitated the operation of the following instruments: a Halo Photonics DWL, the AERI, a Vaisala RS-41 radiosonde ground base station, and a Vaisala WXT530 surface meteorological station (See Ref. 10 for details).

The CLAMPS-2 provided complementary profiles of the ABL which were retrieved though DWL and AERI measurements. The Halo Photonics DWL uses a Plan Position Indicator (PPI) scanning arrangement (i.e., scanning the beam through a full $360^{\circ}$ at a selection of evenly separated azimuth angles at a fixed elevation) combined with the Velocity Azimtuh Display (VAD) algorithm ${ }^{11}$ to estimate the 3D air motion every 3 minutes. During the remaining time, it points vertically and measures the vertical air velocity (VV). The ABLH is estimated by thresholding the VV standard deviation as described in Sect. 3.2. The AERI, on the other hand, is a passive ground-based Fourier transform spectrometer which detects the downwelling infrared radiation from the atmosphere. ${ }^{12}$ These radiation measurements are used to retrieve cloud properties, trace-gases, and profiles of temperature and water vapour by means of the retrieval algorithm called AERI Optimal Estimation (AERIoe). ${ }^{13}$ The estimation of the ABLH is performed combining virtual potential temperature, which is calculated from the AERIoe temperature and moisture profiles, and the parcel method (PM) (See Sect. 3.3).

\section{ABLH RETRIEVAL METHODS}

\subsection{Estimations from the S-band radar by means of the Extended Kalman Filter}

Before the EKF-based CBLH estimation method is applied to the UMass S-band radar reflectivity observations, echoes from nonmeteorological targets (e.g., inserts, birds, and aircraft) are removed by the application of a 
Taula 1. Main specifications of the instruments. (HPBW) Half Power Beam Width. (1) VAD scan from 8 azimuth angles using $60^{\circ}$ elevation. ${ }^{(2)}$ Products retrieved from AERIoe. (3) Height-dependent spatial resolution: $10 \mathrm{~m}$ adjacent to the surface and $300 \mathrm{~m}$ at $3 \mathrm{~km} .{ }^{10}$

\begin{tabular}{|c|c|c|c|c|c|}
\hline Instrument & $\begin{array}{l}\text { Measured } \\
\text { Parameter }\end{array}$ & Pointing & Wavelength & $\begin{array}{l}\text { Raw spatial } \\
\text { resolution }\end{array}$ & $\begin{array}{l}\text { Raw temporal } \\
\text { resolution }\end{array}$ \\
\hline $\begin{array}{l}\text { UMass } \\
\text { S-band radar }\end{array}$ & $\begin{array}{c}\text { Volume } \\
\text { reflectivity } \\
{\left[\mathrm{m}^{-1}\right]}\end{array}$ & $\begin{array}{c}3 \mathrm{deg} \\
(\mathrm{HPBW})\end{array}$ & $\begin{array}{c}10 \mathrm{~cm} \\
(\mathrm{FMCW})\end{array}$ & $5 \mathrm{~m}$ & $16.1 \mathrm{~s}$ \\
\hline $\begin{array}{l}\text { DWL (Halo } \\
\text { Stream Line } \\
\text { XR) }\end{array}$ & $\begin{array}{l}\text { Vertical air } \\
\text { velocity [m } \\
\left.\mathrm{s}^{-1}\right]\end{array}$ & $\begin{array}{c}\text { VAD (1) } \\
\text { Zenith } \\
\text { sampling }\end{array}$ & $1.5 \mu \mathrm{m}$ & $\begin{array}{l}26 \mathrm{~m} \\
30 \mathrm{~m}\end{array}$ & $\begin{array}{l}3 \mathrm{~min} \\
1 \mathrm{~Hz}\end{array}$ \\
\hline $\begin{array}{l}\text { AERI (v4 } \\
\text { MP-3000A) }\end{array}$ & $\begin{array}{c}\text { AERIoe }(2) \text { : } \\
\text { Temperature } \\
{\left[{ }^{\circ} \mathrm{C}\right]} \\
\text { Water Vapor } \\
{\left[\mathrm{g} \mathrm{kg}^{-1}\right]}\end{array}$ & Vertically & $\begin{array}{c}3.3-18.2 \\
\mu \mathrm{m}\end{array}$ & $\begin{array}{c}10-300 \mathrm{~m} \\
(3)\end{array}$ & $5 \min$ \\
\hline
\end{tabular}

time-height $7 \times 7$ median filter to the raw reflectivity image, followed by a 1-dB threshold that generates a binary mask to classify the signals as either "noisy" or çlean", finally retrieving a clean reflectivity image for EKF application. ${ }^{14}$

The EKF is an adaptive algorithm that recursively relates the vertical radar reflectivity profile at the present measurement time with past ABLH estimates as well as measurement noise, atmospheric state vector, and $a$ priori user state vector covariance information in order to yield an optimal ABLH estimate at present time. The EKF algorithm is based on two models: (i) the measurement model, which is an erf-like function parameterization that models the abrupt transition between the CBL and the FA, and (ii) a state-vector transition model, which describes the short-term random transitions of the CBL-to-FA parametrization. The ABLH is estimated as the inflection point of the erf-like model reflectivity profile. Because the EKF yields time-continuous ABLH estimates, it advantageous over non-adaptive classic estimation methods such those based on gradient methods ${ }^{15}$ or a threshold criterion. ${ }^{16}$

\subsection{Estimations from the DWL}

ABLH retrievals from the DWL were obtained by means of the vertical velocity standard deviation (VVSTD) threshold method that consists of establishing a threshold value, $\sigma_{t h}$, to the VV standard deviation, $\sigma_{w}$, and then considering the ABLH as the first height where $\sigma_{w}$ becomes lower than $\sigma_{t h}{ }^{2}$

In this article, we only have available low-temporal resolution measurements of VV from the azimuthal scans performed every $3 \mathrm{~min}$ by the DWL. The valid VV samples are given by the maximum trustable height that is a Carrier-to-Noise Ration (CNR) quality-assured criterion provided by Ref. 17. As an example, in Fig. 1b, the maximum trustable height is computed as the region ensuring a CNR ratio $>-26 \mathrm{~dB}$. The standard deviation is computed every 3 min using a 30-min window. We assumed a threshold $\sigma_{t h}=0.15 \mathrm{~m} \mathrm{~s}^{-1}$, which is based on a model profile of $\sigma_{w}$ by Ref. 18. In order to analyze threshold sensitivity, upper and lower bounds for the ABLH estimates are obtained by applying a perturbation to the nominal threshold, $\sigma_{t h, \pm}=\sigma_{t h} \pm 0.05 \mathrm{~m} \mathrm{~s}^{-1}$. Figure 1c shows the retrieved biases in response to distinct thresholds. We take into account the threshold sensitivity and the error of estimation of $\sigma_{w}$ to estimate the uncertainty of the ABLH estimates provided by the threshold method.

\subsection{Estimations from AERI}

Profiles of virtual potential temperature (VPT, commonly denoted $\theta_{v}$ in meteorological literature) are retrieved from the AERIoe products. The ABLH is estimated by means of the PM, in which the ABLH is taken as the 
(a)

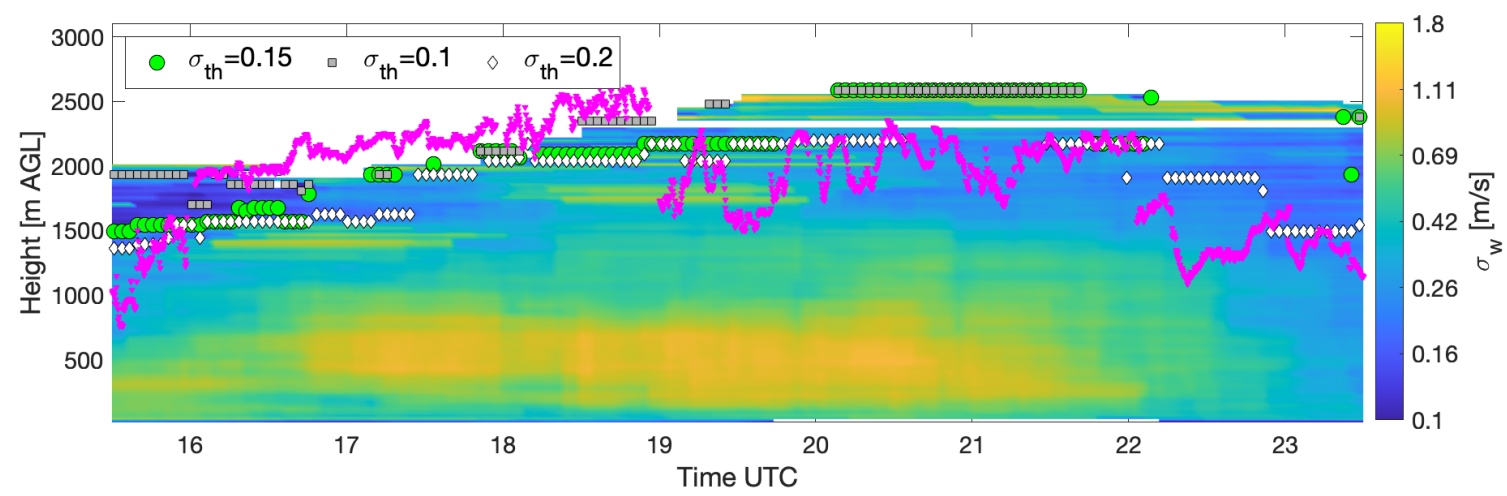

(b)

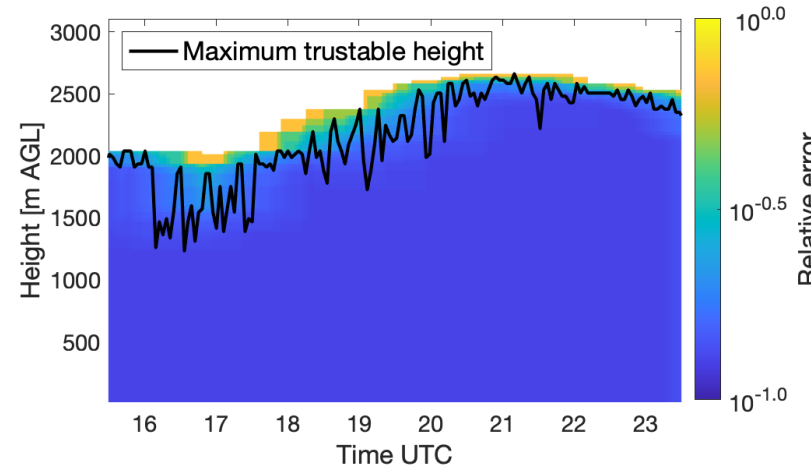

(c)

Figura 1. ABLH estimation based on low-temporal resolution DWL measurements from 16 March 2017. (a) Estimated ABLH associated with different VVSTD thresholds: (green) $\sigma_{t h}=0.15 \mathrm{~m} \mathrm{~s}^{-1},\left(\right.$ grey) $\sigma_{t h}=0.10$ $\mathrm{m} \mathrm{s}^{-1}$, (white) $\sigma_{t h}=0.20 \mathrm{~m} \mathrm{~s}^{-1}$, (pink) ABLH estimated from the EKF-radar combination. The background shading is the estimate of VVSTD from the low temporal resolution $(\Delta t=3 \mathrm{~min})$ DWL data. (b) Filled color plot showing the relative error in the estimate of the VVSTD as function of height and time. The black solid line represents the height of maximum reliable measurements provided by the instrument. ${ }^{17}$ (c) Difference (in meters) between the estimated $\operatorname{ABLH}\left(\sigma_{t h}=0.15 \mathrm{~m} \mathrm{~s}^{-1}\right)$ and $A B L H\left(\sigma_{t h} \pm 0.05 \mathrm{~m} \mathrm{~s}^{-1}\right)$ corresponding to 30 perturbations in the detection threshold. 
height where the VPT is the same that at ground level. ${ }^{19}$ This method is only valid in CBL regime. The AERIoe algorithm provides a error covariance matrix $S$ for its retrieved profiles that must be taken into account when estimating the ABLH. For this reason, $N$ different profiles of virtual potential temperature are generated with a Monte Carlo sampling method, i.e.,

$$
\hat{x}_{i}=S^{1 / 2} z_{i}+x,
$$

where $z_{i}$ is a normally distributed random vector, $x$ is the profile provided by AERIoe and $\hat{x}_{i}$ a new profile within the uncertainty given by $S .{ }^{13}$ From every $\hat{x}_{i}$ an ABLH is retrieved using the PM. The ABLH estimate from AERI is the mean of the $N$ estimations, the standard deviation of all these samples is taken as the uncertainty of the ABLH estimate.

\subsection{ABLH estimation and uncertainty}

ABLH estimates and associated uncertainties are computed according to Eqs. (1-6) in companion paper Ref. 8. In following Sect. 4, key equations are summarised as embedded equations in the text along with cross-references.

\section{DISCUSSION RESULTS}

The 31 March 2017 (Fig. 2) case study is used to compare CBLH estimates from the radar-EKF method, the DWL-VVSTD method, and AERI-PM method. Local time is LT $=$ UTC -5 h. In Fig. 2b, hourly estimates of the ABLH are computed as the maximum likelihood mean (Eq. 1 in Ref. 8) over intervals of 60 minutes. The ABLH-hourly uncertainties (error bars in Fig. 2b) of a retrieval method "x" (EKF, DWL, and AERI) are estimated by means of the law of error superposition (Ref. 20, Eq. 4.14), formally, $\sigma_{x}=\sqrt{\sigma_{x 1}^{2}+\sigma_{x 2}^{2}}$. The variable $\sigma_{x 1}$ is the standard deviation computed hourly, and represents the atmospheric variability of the ABLH-hourly estimates, whereas $\sigma_{x 2}$ is the uncertainty error associated with ABLH-hourly, $\sigma_{x 2}=\sqrt{1 / \sum \sigma_{i}^{2}}$, where $\sigma_{i}$ is the instrumental error for a time $t_{i}$ (Eqs. 2-4, therein). Finally, Fig. 2c shows the ABLH bias as function of time between different ABLH estimates. The uncertainty of the ABLH bias is also obtained by the law of error superposition, now taking into account the ABLH-hourly uncertainties of each of the ABLH estimates used to compute the ABLH bias. The contents of this paragraph are explained in detail in the companion paper, Ref. 8.

During the convective period (1400 - 2300 UTC), in which the CBL is the most turbulent, the CBLH reaches its maximum (Fig. 2 a, b). Inside this period, the CBLH estimates by radar-EKF and the DWL-VVSTD show the best agreement, as can be seen in Fig. $2 \mathrm{~b}$ and $2 \mathrm{c}$; the ABLH biases between radar-EKF and DWL-VVSTD ABLH estimates are between -100 and $100 \mathrm{~m}$. In Fig. 2a, many missing CBLH estimates from the DWL threshold method can be seen; these are a result of using DWL-VVSTD estimates based on low-temporal resolution (3 min) DWL data.

The AERI-PM method consistently underestimates the ABLH by approximately $1000 \mathrm{~m}$ (Fig. 2a). This unexpected result is still under study. From the point of view of Information Theory, the largest amount of information used in the AERIoe retrieval is concentrated in the lower hundreds of meters of the atmosphere, and decreases exponentially with height. The reduced resolution of the AERIoe temperature and moisture profiles above 1000 m may partly explain this poor ABLH retrieval.

"Global" averages (i.e., arithmetic means of all CBLH hourly estimates) are computed for the 2017 VORTEXSE campaign for 19 clear-sky, non-precipitation case days. In each, ABLH hourly estimates are computed for each of the distinct CBLH-retrieval methods (Fig. 3a). Fig. 3b shows the intercomparison between the globally averaged ABLH biases. Both plots are focused on the CBL period (1400 - 2300 UTC). These results confirm generally what has been discussed previously for the particular case of Fig. 2:

1. The AERI-PM is not providing reliable ABLH estimates above $1000 \mathrm{~m}$.

2. When comparing the ABLH retrievals from DWL and the EKF-radar there is almost zero bias when the ABLH is fully developed (1700 UTC to 2200 UTC). This bias increases during the transition times (near sunrise and sunset). 
(a)

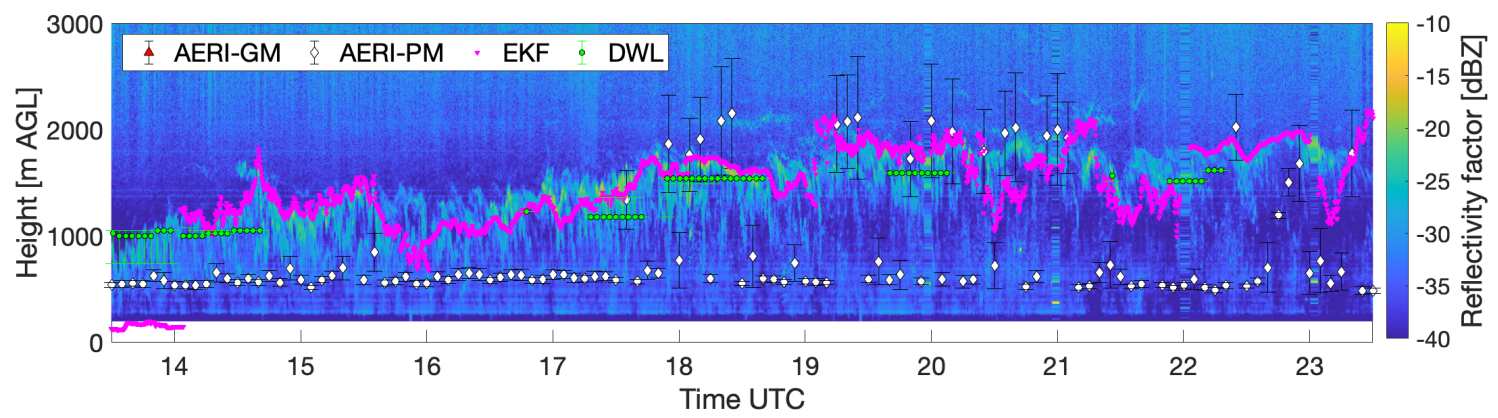

(b)

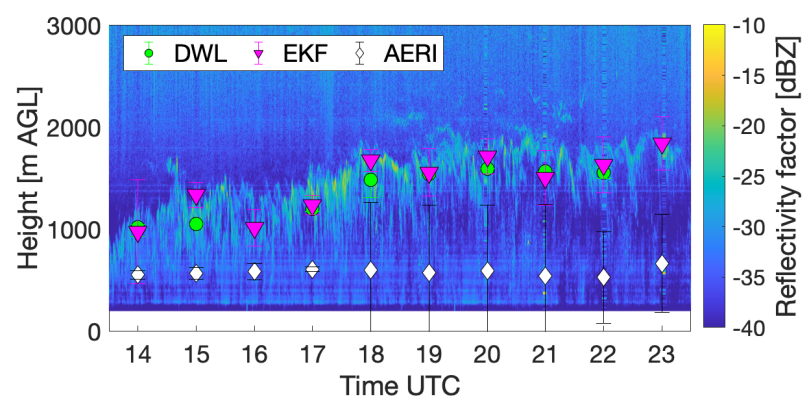

(c)

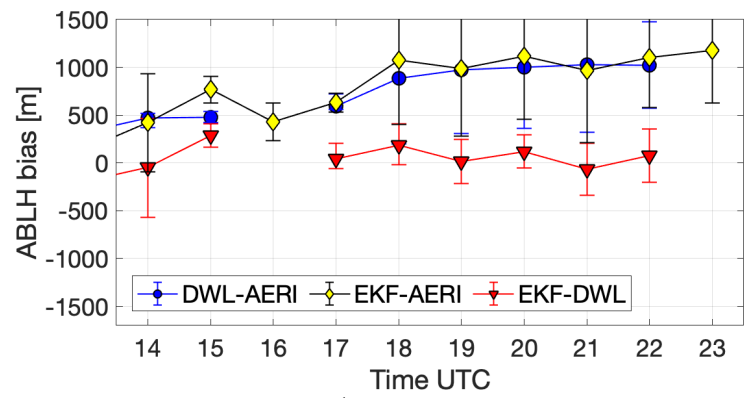

Figura 2. (a) ABLH (in m) estimated over the diurnal cycle of a clear day (0700 UTC 16 April 2017 to 0600 UTC 17 April 2017; LT = UTC-5h) from (magenta triangles) ABLH-radar, (green dots) ABLH-DWL, (white diamonds) ABLH-AERI PM, and (red triangles) ABLH-AERI GM in the stable boundary layer. Also plotted are radar reflectivity factor (color fill, in dBZ), and cloud-base height measured by the Vaisala ceilometer (yellow dots, in $\mathrm{m}$ ). (b) As in (a), but plotted as 1-h averages as function of time. Markers indicate the estimated value; error bars indicate the $1 \sigma$ uncertainty. (c) 1-h ABLH bias as function of time for (blue) DWL minus AERI, (yellow) EKF minus AERI, and (red) EKF minus DWL. Markers indicate the estimated value; error bars indicate the $1 \sigma$ uncertainty.

As previously explained, the EKF-radar ABLH estimate tends to follow the RL, especially in the afternoon-toevening transition, while the DWL-VVSTD ABLH estimates strands at very low heights when the turbulence weakens.

Fig. 4 shows histograms of the ABLH bias between the DWL-VVSTD and the EKF-radar estimates computed hourly for the 19-day sample. At the middle of the convective period (1700 UTC to 2000 UTC), most of the counts are concentrated in the 200 to $400 \mathrm{~m}$ bins, denoting a systematic positive bias in the ABLH estimates from the EKF-radar method versus the DWL-VVSTD method. During transition times (i.e., 1300-1400 UTC and 2100-2300 UTC), the counts are scattered over a wider range of bins, denoting greater disagreement among the ABLH estimates. The ABL structure is more variable during transition times, which we hypothesize leads to greater uncertainty in the ABLH estimates. The reader is reminded that we are restricted to using DWL low-temporal resolution data, which has been proven to yield less certain estimates of ABLH than high resolution DWL data. ${ }^{8}$ Accordingly, many outliers fall outside of $\mu \pm 1-\sigma$ confidence bounds.

\section{CONCLUSION}

The EKF was applied to UMass S-band radar returns to obtain automatic and time-continuous CBLH tracking, using data from the VORTEX-SE 2017 field campaign. Radar-EKF performance was investigated against CBLH from DWL observations, retrieved by a standard deviation threshold method, and CBLH estimated from AERI, retrieved by means the parcel method. Based on a case study (31 March 2017) and the global statistics based on 19 days of clear-sky observation, it was shown that during the periods of the day when the CBL is highly developed, the CBLH estimates from radar-EKF retrieved results are similar to those estimated from DWL-VVSTD method. 
(a)

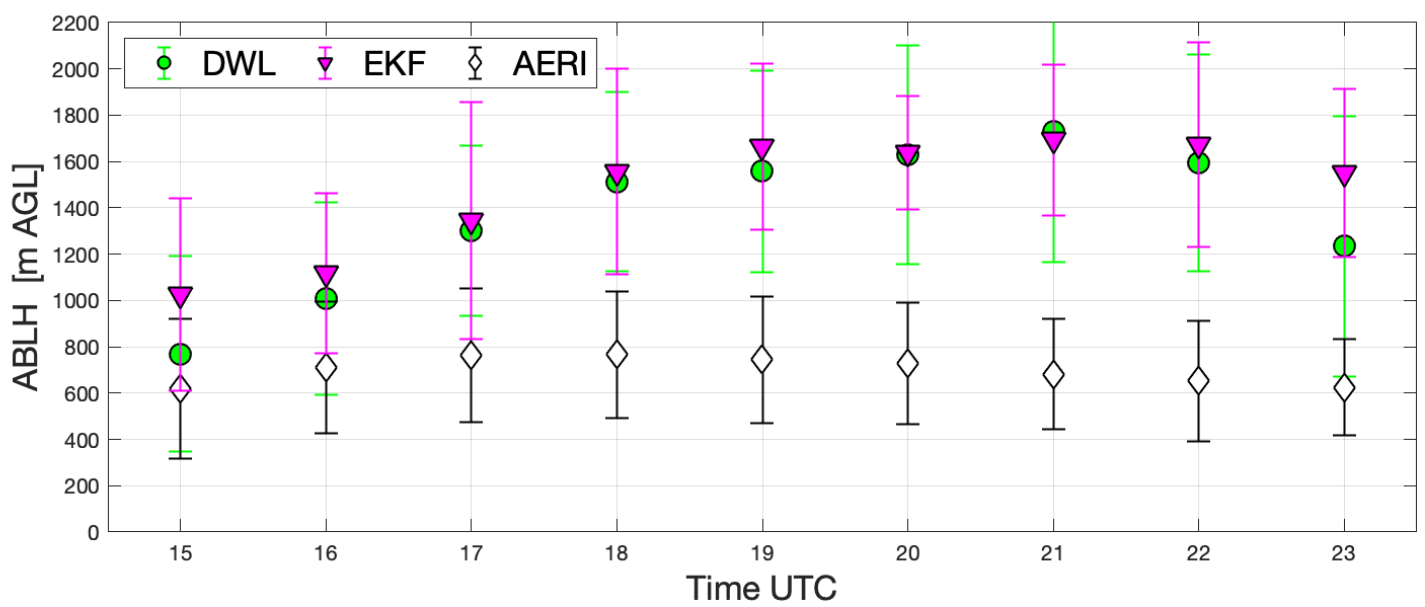

(b)

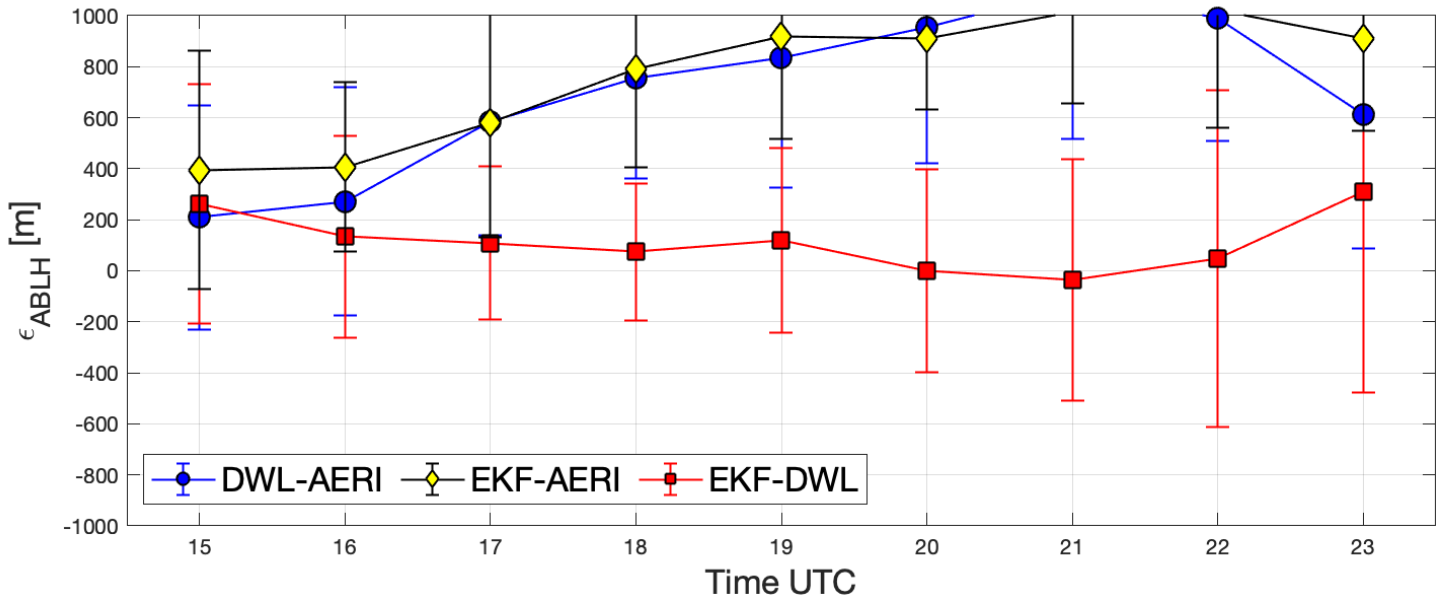

Figura 3. Hourly ABLH estimates and biases averaged over the selected 19 clear-sky case days as function of hour in UTC (LT = UTC-5h). (a) ABLH estimate mean values from (magenta triangles) ABLH-radar, (green dots) ABLH-DWL, (white diamonds) and ABLH-AERI, and standard deviations (associated error bars). (b) ABLH bias mean values for (blue) $A B L H_{D W L}\left(t_{h}\right)-A B L H_{A E R I}\left(t_{h}\right)$, (yellow) $A B L H_{\text {radar }}\left(t_{h}\right)-A B L H_{A E R I}\left(t_{h}\right)$, and (red) $A B L H_{\text {radar }}\left(t_{h}\right)-A B L H_{D W L}\left(t_{h}\right)$. Error bars indicate standard deviations. 


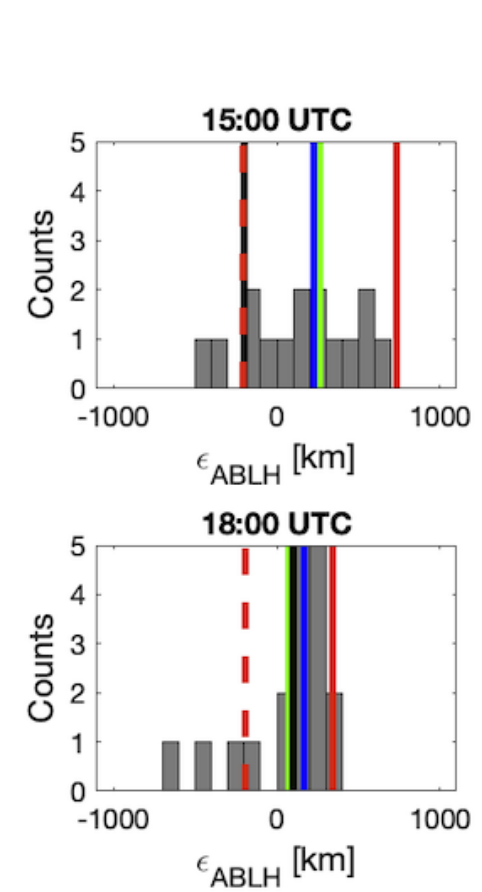

$\mathrm{ABLH}_{E K F}-\mathrm{ABLH} \mathrm{DWL}_{\mathrm{DW}}$
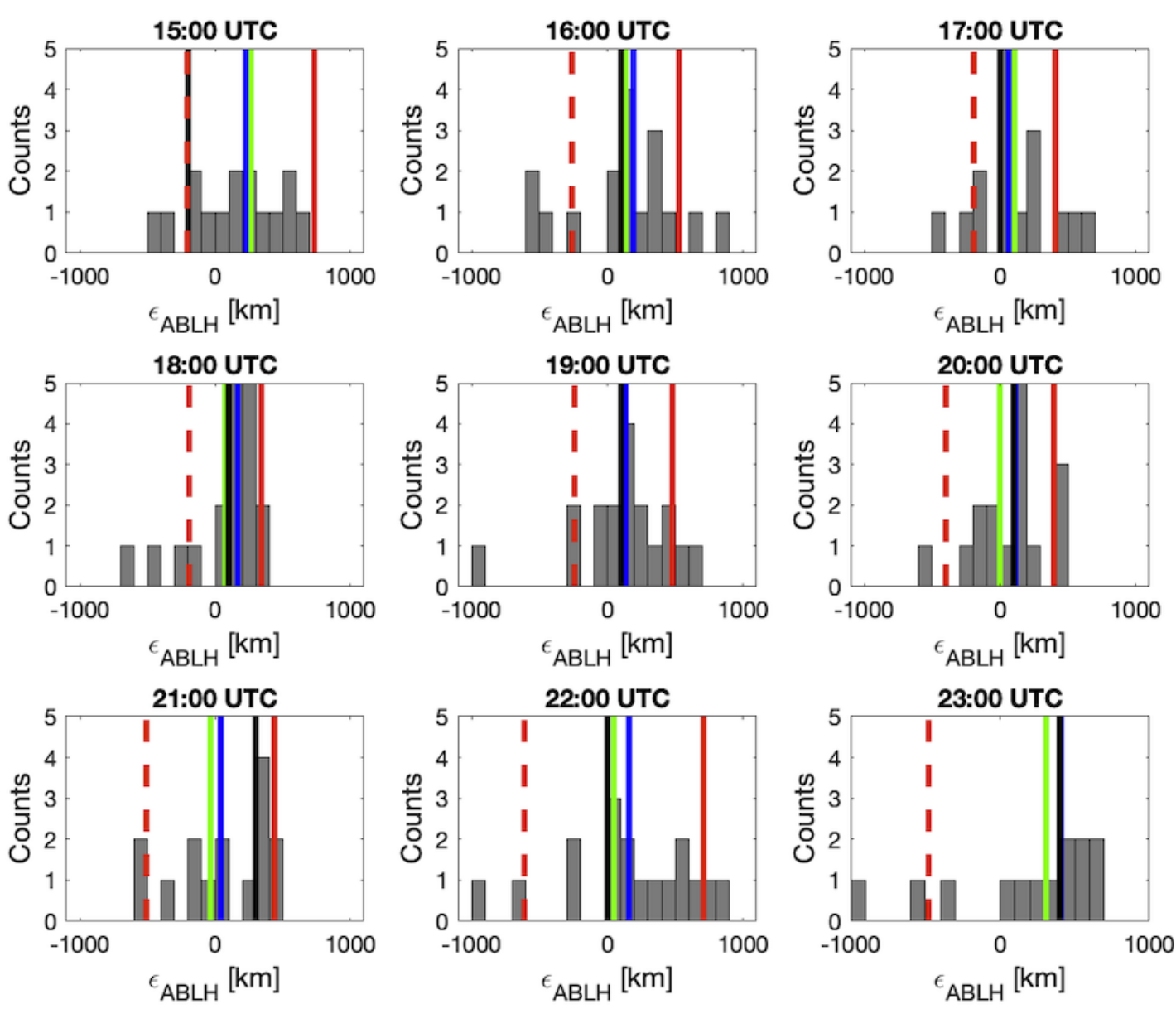

\section{Mean —Median —Mode $-\mu+\sigma--\mu-\sigma$}

Figura 4. Histograms of the bias (in $\mathrm{m}$ ) between the DWL and the EKF-radar ABLH estimates for the selected 19 clear-sky case days. Shown are (green line) the mean, (blue line) median, (black line) mode, and the 1- $\sigma$ distances from the mean value $(\mu ; \mu+\sigma$ red solid line, and $\mu-\sigma$ red dashed line). 
However, larger differences were found during morning and afternoon transition times. Additionally, in the case study, the EKF tends to track the residual layer during the morning transition time. When comparing CBLH estimates from radar-EKF to DWL-VVSTD, the differences over transition periods were higher due to the DWLVVSTD-method's dependence on turbulence intensity. We also conclude that the AERI-PM method substantially underestimates CBLH (by a factor of 2 or more) due to relative the lack of information in the AERIoe profiles over $1000 \mathrm{~m}$, which results in unrealistic CBLH estimates.

The 19-day data subset studied in the context of the 2017 VORTEX-SE campaign (8 March to 8 May 2017; Alabama, U.S.A.) has yielded key strengths and weaknesses (SaW; Tab. 2) for the various combinations of remote-sensing instruments and ABLH retrieval methods, some of which are dependent on the stage of the diurnal ABL cycle. Although limited to clear days only, the SaW table provides the grounds to tackle a larger sample of data (both 2016 and 2017 field campaigns) as well as defining data processing guidance.

Taula 2. Summary of strengths and weaknesses for each remote-sensing instrument and its associated ABLH retrieval methods.

\begin{tabular}{|c|c|c|c|}
\hline Instrument & Method & Strengths & Weaknesses \\
\hline $\begin{array}{l}\text { UMass S-band } \\
\text { radar }\end{array}$ & $\mathrm{EKF}$ & $\begin{array}{l}\text { - High temporal and spatial } \\
\text { resolution. } \\
\text { - Direct measurement of the } \\
\text { structure parameter for the } \\
\text { refractive index, } C_{n}^{2} \text {. } \\
\text { - Reliable ABLH estimation in } \\
\text { CBL regime. }\end{array}$ & $\begin{array}{l}\text { - Low signal at night and in } \\
\text { morning transition time. } \\
\text { - Detection of the RL in the } \\
\text { afternoon transition time. } \\
\text { - Very large files (1h-400 MB). }\end{array}$ \\
\hline $\begin{array}{l}\text { DWL (Halo } \\
\text { Stream Line XR) }\end{array}$ & $\begin{array}{l}\text { Threshold } \\
\text { method LR }\end{array}$ & $\begin{array}{l}\text { - Measurement of turbulence. } \\
\text { - Reliable ABLH estimation in } \\
\text { CBL regime. }\end{array}$ & $\begin{array}{l}\text { - High VVSTD threshold } \\
\text { sensitivity. } \\
\text { - Height range limited by the } \\
\text { aerosol concentration. }\end{array}$ \\
\hline $\begin{array}{l}\text { AERI (v4 } \\
\text { MP-3000A) }\end{array}$ & Parcel Method & $\begin{array}{l}\text { - Based on thermodynamic } \\
\text { properties of the ABL. }{ }^{3}\end{array}$ & $\begin{array}{l}\text { - Low temporal end spatial } \\
\text { resolution. } \\
\text { - Degraded information content } \\
\text { and resolution at altitudes above } \\
1000 \mathrm{~m} \text {. } \\
\text { - Consistently underestimated } \\
\text { ABLH in CBL in this study. }\end{array}$ \\
\hline
\end{tabular}

\section{Acknowledgements}

This work was supported by NOAA grants NA1501R4590232 and NA16OAR4590209, and by the Purdue University Dept. of Earth, Atmospheric, and Planetary Sciences (EAPS). CommSensLab-UPC (Excellence Unit MDM-2016-0600 funded by the Agencia Estatal de Investigación, Spain) collaborated via Spanish Gov. - EU Regional Development Funds, ARS project PGC2018-094132-B-I00 and ACTRIS-PPP project GA-739530. The Spanish National Science Foundation (Ministerio de Ciencia, Innovación y Universidades) funded doctoral grant PRE2018-086054 hold by M.P. Araujo da Silva. The University of Massachusetts, Microwave Remote Sensing Laboratory and EAPS deployed the mobile S-band radar, co-located Vaisala CL-31 ceilometer, and portable disdrometers. The NOAA National Severe Storms Laboratory deployed the Collaborative Lower Atmosphere Mobile Profiling System. The statements, findings, conclusions, and recommendations are those of the authors and do not necessarily reflect the views of the NOAA or the U.S. Department of Commerce. 


\section{REFERENCES}

[1] Lange, D., Rocadenbosch, F., Tiana-Alsina, J., and Frasier, S., "Atmospheric boundary layer height estimation using a kalman filter and a frequency-modulated continuous-wave radar," IEEE Transactions on Geoscience and Remote Sensing 53(6), 3338-3349 (2015).

[2] Schween, J. H., Hirsikko, A., Löhnert, U., and Crewell, S., "Mixing-layer height retrieval with ceilometer and doppler lidar: from case studies to long-term assessment," Atmospheric Measurement Techniques 7(11), 3685-3704 (2014).

[3] Stull, R. B., [An Introduction to Boundary Layer Meteorology], Kluwer Academic Publishers (1988).

[4] Wallace, J. M. and Hobbs, P. V., [Atmospheric science: an introductory survey], vol. 92, Elsevier (2006).

[5] NOAA/DOC, "VORTEX Southeast," (2016).

[6] Tanamachi, R. L., Frasier, S. J., Waldinger, J., LaFleur, A., Turner, D. D., and Rocadenbosch, F., "Progress toward characterization of the atmospheric boundary layer over northern alabama using observations by a vertically pointing, s-band profiling radar during vortex-southeast," Journal of Atmospheric and Oceanic Technology 36(11), 2221-2246 (2019).

[7] Lange, D., Tiana-Alsina, J., Saeed, U., Tomás, S., and Rocadenbosch, F., "Atmospheric boundary layer height monitoring using a kalman filter and backscatter lidar returns," IEEE Transactions on Geoscience and Remote Sensing 52(8), 4717-4728 (2014).

[8] Rocadenbosch, F., Tanamachi, R. L., Araujo da Silva, M. P., Villalonga, J., Frasier, S. J., and Turner, D. D., "Convective boundary-layer height estimation from combined radar and doppler lidar observations in vortex-se atmospheric boundary layer height disambiguation using synergetic remote sensing observations: case examples from vortex-se," (2020). Proc. SPIE 11531, Remote Sensing of Clouds and the Atmosphere XXV, 11531-19 (22 September); In print.

[9] İnce, T., Frasier, S. J., Muschinski, A., and Pazmany, A. L., "An s-band frequency-modulated continuouswave boundary layer profiler: Description and initial results," Radio Science 38(4) (2003).

[10] Wagner, T. J., Klein, P. M., and Turner, D. D., "A new generation of ground-based mobile platforms for active and passive profiling of the boundary layer," Bulletin of the American Meteorological Society 100(1), 137-153 (2019).

[11] Fujii, T. and Fukuchi, T., [Laser Remote Sensing], CRC Press (2005).

[12] Knuteson, R., Revercomb, H., Best, F., Ciganovich, N., Dedecker, R., Dirkx, T., Ellington, S., Feltz, W., Garcia, R., Howell, H., Smith, W., and Short, J., "Atmospheric emitted radiance interferometer. part i: Instrument design," Journal of Atmospheric and Oceanic Technology - J ATMOS OCEAN TECHNOL $\mathbf{2 1}$ (12 2004).

[13] Turner, D. D. and Löhnert, U., "Information content and uncertainties in thermodynamic profiles and liquid cloud properties retrieved from the ground-based atmospheric emitted radiance interferometer (aeri)," Journal of Applied Meteorology and Climatology 53(3), 752-771 (2014).

[14] Contreras, R. F. and Frasier, S. J., "High-Resolution Observations of Insects in the Atmospheric Boundary Layer," Journal of Atmospheric and Oceanic Technology 25(12), 2176-2187 (2008).

[15] Endlich, R., Ludwig, F., and Uthe, E., "An automatic method for determining the mixing depth from lidar observations," Atmospheric Environment (1967) 13(7), 1051 - 1056 (1979).

[16] Melfi, S. H., Spinhirne, J. D., Chou, S.-H., and Palm, S. P., "Lidar observations of vertically organized convection in the planetary boundary layer over the ocean," Journal of Climate and Applied Meteorology 24(8), 806-821 (1985).

[17] Turner, D., "Ou/nssl clamps doppler lidar wind profile data. version 1.0," (2017).

[18] Lenschow, D. H., Wyngaard, J. C., and Pennell, W. T., "Mean-field and second-moment budgets in a baroclinic, convective boundary layer," Journal of the Atmospheric Sciences 37(6), 1313-1326 (1980).

[19] Holzworth, G. C., "Estimates of mean maximum mixing depths in the contiguous united states," Monthly Weather Review 92(5), 235-242 (1964).

[20] Barlow, R. J., [Statistics: A Guide To The Use of Statistical Methods in the Physical Sciences], John Wiley \& sons (1989). 